\title{
HuR and podoplanin expression is associated with a high risk of malignant transformation in patients with oral preneoplastic lesions
}

\author{
UMMA HABIBA $^{1}$, TETSUYA KITAMURA ${ }^{1}$, AYA YANAGAWA-MATSUDA ${ }^{1}$, FUMIHIRO HIGASHINO ${ }^{1}$, \\ KYOKO HIDA $^{2}$, YASUNORI TOTSUKA ${ }^{3}$ and MASANOBU SHINDOH $^{1}$ \\ ${ }^{1}$ Department of Oral Pathology and Biology, Hokkaido University Graduate School of Dental Medicine, Sapporo, \\ Hokkaido 060-8586; ${ }^{2}$ Department of Vascular Biology, Hokkaido University Institute for Genetic Medicine, \\ Sapporo, Hokkaido 060-0815; ${ }^{3}$ Department of Oral and Maxillofacial Surgery, \\ Hokkaido University Graduate School of Dental Medicine, Sapporo, Hokkaido 060-8586, Japan
}

Received November 17, 2014; Accepted January 12, 2016

DOI: 10.3892/ol.2016.5061

\begin{abstract}
The risk of malignant transformation in oral preneoplastic lesions (OPLs) is challenging to assess. The objective of the present study was to determine the expression of ELAV like RNA binding protein 1 (HuR) and podoplanin in OPLs, and to evaluate the use of each protein as biomarkers for the risk assessment of malignant transformations. Immunohistochemistry for HuR and podoplanin was performed on the tissues of 51 patients with OPL, including cases of low grade dysplasia (LGD) and high grade dysplasia (HGD). The association between the protein expression patterns and clinicopathological parameters, including oral cancer free survival (OCFS) time, was analyzed during the follow-up period. HuR and podoplanin expression was observed in $28(55 \%)$ and $36(71 \%)$ of 51 patients, respectively. Kaplan-Meier analysis showed that the expression of HuR and podoplanin was associated with the risk of progression to oral cancer $(\mathrm{P}<0.05)$. Multivariate analysis revealed that $\mathrm{HuR}$ and podoplanin expression was associated with a 2.93-fold (95\% confidence interval (CI), 0.98-10.34; $\mathrm{P}=0.055$ ) and 2.06-fold (95\% CI, 0.55-8.01; $\mathrm{P}=0.283)$ increase in risk of malignant transformation, respectively. The risk of OPL malignant transformation was considerably increased with the coexpression of $\mathrm{HuR}$ and podoplanin compared with the histological grading ( $95 \% \mathrm{CI}, 1.64-23.59 ; \mathrm{P}=0.005)$. The results of the present study demonstrated that the expression of $\mathrm{HuR}$ and podoplanin associates with malignant
\end{abstract}

Correspondence to: Professor Masanobu Shindoh, Department of Oral Pathology and Biology, Hokkaido University Graduate School of Dental Medicine, North 13, West 7, Sapporo, Hokkaido 060-8586, Japan

E-mail: mshindoh@den.hokudai.ac.jp

Key words: oral preneoplastic lesion, malignant transformation, HuR, podoplanin transformation and suggests that the proteins may be used as biomarkers to identify OPL patients with an increased risk of cancer development.

\section{Introduction}

Oral epithelial dysplasia (OED) is the diagnostic histopathological term used to describe an oral preneoplastic lesion (OPL), and is predictive of an increased rate of developing oral squamous cell carcinoma (OSCC) (1). However, assessing the risk of the malignant transformation of OPLs is challenging. Early diagnosis of high-risk, potentially malignant lesions is a high priority for reducing morbidity and mortality (1-3). Studies with a median follow-up of $>7$ years have reported malignant transformation rates between 17 and 20\% (4). Although lesions with dysplastic features are considered to be at an increased risk for malignant transformation, the majority of the oral cancers develop from lesions that lack dysplastic changes $(4,5)$. Therefore, objective biomarkers are required to evaluate the risk of malignant transformation in OPL, and for the prophylactic intervention and proper management of high risk patient groups.

ELAV like RNA binding protein 1 (HuR) is an ubiquitously expressed mRNA-binding protein. Intracellularly, HuR is localized predominantly in the nucleus, but shuttles between the nucleus and the cytoplasm (6). The export of HuR is mediated by the association with transportin 1 and 2, via the HuR nucleocytoplasmic shuttling sequence in the hinge region, and the association with the acidic nuclear phosphoprotein 32 family member $\mathrm{A}$, a proliferation-inducing ligand and the SET nuclear proto-oncogene $\alpha / \beta$ protein, which includes the nuclear export signal recognized by the export receptor chromosome maintenance region 1 (6-8). AU-rich elements (ARE) are located in the untranslated regions of numerous proto-oncogenes, growth factors and cytokine mRNAs as the core sequence of AUUUA. HuR binds to AREs to protect ARE-mRNAs against rapid degradation. As nucleocytoplasmic translocation is necessary for the activity of $\mathrm{HuR}$ and the cytoplasmic presence of HuR is indicated in 
several carcinomas, cytoplasmic HuR expression is hypothesized to be a prognostic marker in cancer patients $(9,10)$.

Podoplanin is a mucin-type transmembrane glycoprotein that is specifically expressed in lymphatic endothelial cells, but not in blood endothelial cells (11). Podoplanin has been identified as a potential marker for the progression of oral leukoplakia to invasive carcinoma (12-14). Wicki and Christofori (15) suggested that podoplanin may act as a mediator of tumor cell invasion and metastasis. Podoplanin is also expressed in the hyperplastic and dysplastic regions that are adjacent to primary tumors, which indicates that the abnormal expression of podoplanin occurs early in oral tumorigenesis (16). In addition, in oral premalignant lesions certain molecular genetic traits are in common with OSCC, even in the absence of histologically defined dysplasia (17). Therefore, the purpose of the present study was to investigate the potential association between HuR and podoplanin expression in OPL, with or without malignant transformation, and to determine the usefulness of the proteins as biomarkers for cancer risk assessment.

\section{Materials and methods}

Patients and tissue specimens. All medical records of the 51 patients that were diagnosed with OPL between March 2001 and May 2012 at Hokkaido University Hospital (Sapporo, Japan) were retrieved and reviewed at the Department of Oral Pathology and Biology, Hokkaido University Graduate School of Dental Medicine (Sapporo, Japan). The clinical data were obtained from the medical records and biopsy specimens were obtained from formalin-fixed paraffin-embedded tissues. The expression of HuR and podoplanin were determined in 51 patients with OPL during the follow-up period using immunohistochemistry. Associations between the protein expression patterns and clinicopathological parameters, including oral cancer development, during the follow-up were analyzed statistically. In the present retrospective follow-up study, malignant transformation vs. nontransformation was considered as the surrogate for the clinical outcome of patients with OPL. Of the 51 patients with a median follow-up of 55 months, 24 patients (47\%) developed OSCC. The present study was approved by the institutional review board.

Tissue processing and immunohistochemistry. Serial tissue sections ( $5 \mu \mathrm{m}$ thick) from formalin-fixed, paraffin-embedded tissue blocks of OPL were mounted on positively charged glass slides. Immunohistochemical staining was performed using the streptavidin-peroxidase methods, as previously described (18). In brief, sections were deparaffinized in xylene, rehydrated in graded alcohol and subjected to antigen retrieval by heat treatment in Tris-ethylenediaminetetraacetic acid (TE) buffer. In order to inhibit endogenous peroxidase activity, the slides were then immersed in 3\% hydrogen peroxide (Sigma-Aldrich, St. Louis, MO, USA) for $5 \mathrm{~min}$, followed by blocking solution [1\% bovine serum albumin (Sigma-Aldrich) in phosphated-buffered saline (PBS)] for $30 \mathrm{~min}$. The immunohistochemical detection of HuR and podoplanin were performed using anti-HuR (mouse immunoglobulin G anti-human; clone 3A2; dilution, 1:5,000; catalog no., sc-5261; catolog no., sc-5261; Santa Cruz Biotechnology, Inc., Dallas, TX, USA) and anti-podoplanin (mouse anti-human; clone, D2-40; dilution, 1:100; catalog no., M3619;
Vector Laboratories, Inc., Burlingame, CA, USA) monoclonal antibodies, respectively, in blocking solution in a humidified chamber at $4^{\circ} \mathrm{C}$ overnight. The sections were then subjected to Simple Stain Max PO (M) (Nichirei Bioscience, Tokyo, Japan) at $37^{\circ} \mathrm{C}$ for $30 \mathrm{~min}$. Carefully, rinses were performed with several changes of PBS between the stages of the procedure $(5$ minute washes repeated 3 times). Visualization was performed using the ChemMate EnVision kit/HRP (Dako North America, Inc., Carpinteria, CA, USA). Cytoplasmic and cell membrane immunoreactivity in the epithelium was considered to indicate the reaction of HuR and podoplanin, respectively.

In order to analyze the prognostic values for cancer development, the immunoreactivity of HuR and podoplanin was classified into two categories (Figs. 1 and 2). According to the criteria that were described in a previous study (18), the distribution of the HuR protein in the cell was categorized into the three levels of the epithelium: Level 1, lower one-third; level 2, lower two-thirds; and level 3, extending to the upper one-third of the epithelium. A sample evaluation of the immunoreactivity of podoplanin was performed according to the criteria based on staining score, previously described by Kawaguchi et al (14). In accordance with these criteria, scores between 0-4 were determined. No expression resulted in the score 0 , podoplanin expression limited to the basal layer was scored 1, expression in the basal layer and suprabasal layers in one region was scored 2 and podoplanin expression at 2-3 suprabasal regions was scored 3. Podoplanin expression observed in $>3$ areas in the suprabasal layer was scored 4 . The scores were based on the examination of the entire section in each biopsy. Lesions that were classified with level 2 and 3 distributions (positive expression up to lower two-thirds and upper one-third, respectively) were considered to express HuR, and lesions with scores of $\geq 2$ (positive expression in the suprabasal layer in 1 or more areas) were considered to express podoplanin. Accordingly, 28 (55\%) and $36(71 \%)$ of lesions were considered to express HuR and podoplanin, respectively, and the remaining $23(45 \%)$ and $15(29 \%)$ were not.

Statistical analysis. Associations between HuR and podoplanin expression and the clinicopathological variables were assessed using the Wilcoxon rank-sum test for continuously distributed variables and the $\chi^{2}$ test for categorical variables. The Kaplan-Meier survival analysis was used to investigate the associations with oral cancer-free survival (OCFS) time, which is the time interval between the histopathological diagnosis and the development of OSCC. Patients that did not develop invasive OSCC were censored at the final date of follow-up. The log-rank test was used to compare survival times among patients with different characteristics. The Cox proportional hazards regression model was applied to evaluate the hazard ratio (HR) for the malignant transformation of OPLs. HRs with a 95\% confidence interval (CI) and the P-values were reported. All tests were two sided, and P-values of $<0.05$ were considered to indicate a statistically significant difference. The JMP ${ }^{\circledR}$ Pro version 10.0.2 (SAS Institute Inc., Cary, NC, USA) was used for statistical analysis.

\section{Results}

Patient characteristics and HuR and podoplanin expression. The present study comprised of 51 patients, of which 

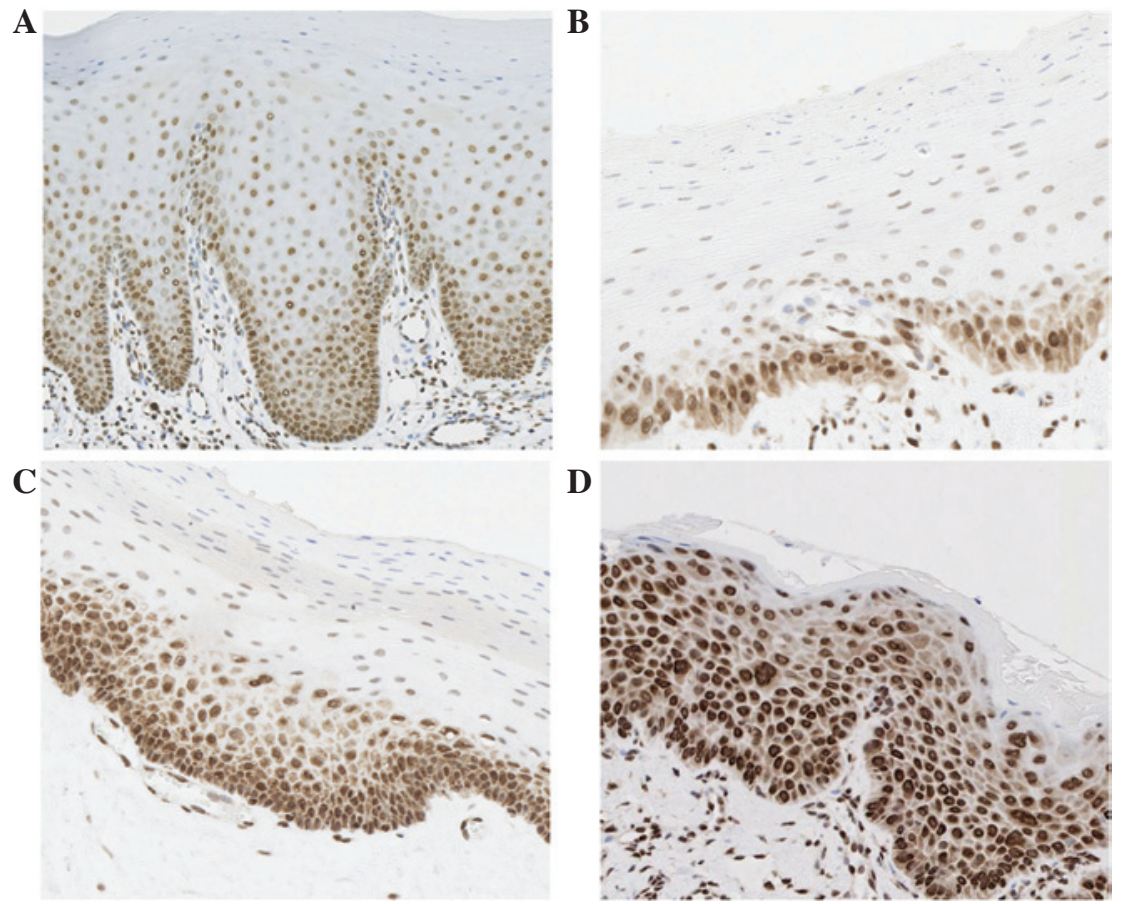

Figure 1. Immunohistochemical analysis of HuR expression. (A) In the normal epithelium, HuR is localized predominantly in the nucleus (magnification, x10). HuR expression in the (B) lower one-third, (C) lower two-third and (D) upper one-third of the epithelium was categorized as level 1,2 and 3, respectively (magnification, $\mathrm{x} 20$ ).
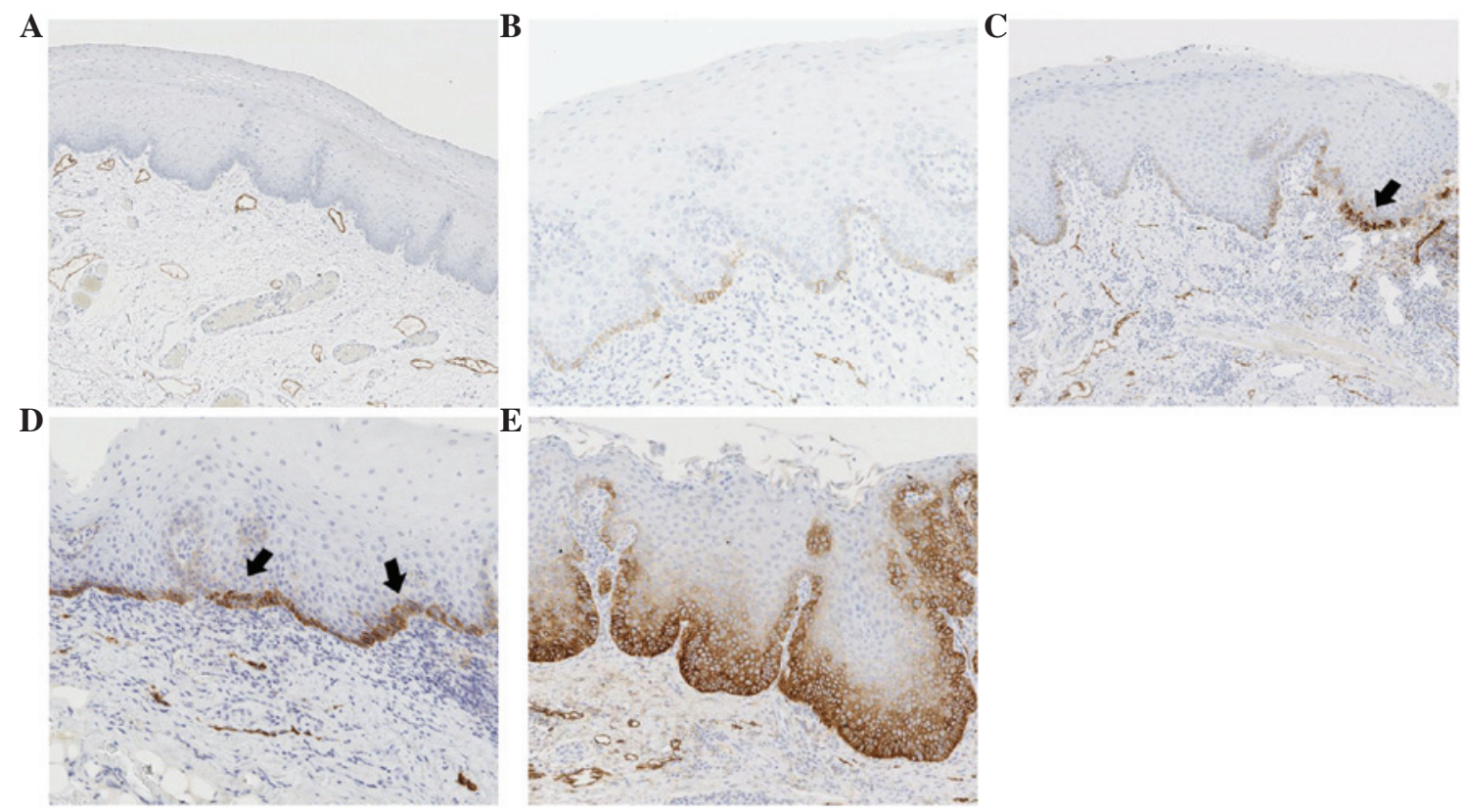

Figure 2. Representative expression of podoplanin. Scores of (A) 0 and (B) 1 were considered to indicate no podoplanin expression and scores of (C) 2 , (D) 3 and (E) 4 were considered to indicate podoplanin expression (magnification, x10). The arrows indicate nests with podoplanin expression.

39 were female and 12 were male. HuR and podoplanin was not expressed in $45 \%$ and $29 \%$ of the patients, whereas expression was observed in $55 \%$ and $71 \%$ of the patients, respectively (Table I). The expression patterns of HuR and podoplanin in serial tissue samples of OPLs are shown in Figs. 1 and 2. With regards to HuR, 23 (45.1\%), 19 (37.3\%) and 9 (17.6\%) cases of OPL showed level 1, level 2 and level 3 expression within the epithelium, respectively; for podoplanin expression, $5(9.8 \%)$ samples were scored as 0, $10(19.6 \%)$ were scored as 1, $9(17.6 \%)$ were scored as 2, $6(11.8 \%)$ were scored as 3 and $21(41.2 \%)$ were scored as 4.

The association between HuR and podoplanin expression and the clinicopathological parameters are summarized in Table II. HuR and podoplanin expression was significantly associated with grades of dysplasia $(\mathrm{P}<0.05)$. While 71 and $82 \%$ of OPLs with HGD were associated with HuR and podoplanin expression, 29 and $18 \%$ of the patients with HGD were not associated with HuR and podoplanin expression, respectively. 
Table I. Patient baseline characteristics.

\begin{tabular}{|c|c|}
\hline Characteristic & No. of patients ( $\%$ \\
\hline All patients & $51(100.0)$ \\
\hline \multicolumn{2}{|l|}{ Age, years } \\
\hline Mean \pm SD & $70.9 \pm 10.2$ \\
\hline Median & 72 \\
\hline \multicolumn{2}{|l|}{ Gender } \\
\hline Female & $39(76.5)$ \\
\hline Male & $12(23.5)$ \\
\hline \multicolumn{2}{|c|}{ Follow-up, months } \\
\hline Mean \pm SD & $42.2 \pm 35.6$ \\
\hline Median & 43 \\
\hline \multicolumn{2}{|l|}{ Site } \\
\hline Tongue & $21(41.2)$ \\
\hline Gingiva & $8(15.7)$ \\
\hline $\mathrm{BM}$ & $14(27.5)$ \\
\hline FOM & $4(7.8)$ \\
\hline Others & $4(7.8)$ \\
\hline \multicolumn{2}{|l|}{ Dysplasia } \\
\hline LGD & $17(33.3)$ \\
\hline HGD & $34(66.7)$ \\
\hline \multicolumn{2}{|c|}{ HuR expression } \\
\hline Level 1 & $23(45.1)$ \\
\hline Level 2 & $19(37.3)$ \\
\hline Level 3 & 9 (17.6) \\
\hline \multicolumn{2}{|c|}{ Podoplanin expression } \\
\hline Score 0 & $5(9.8)$ \\
\hline Score 1 & $10(19.6)$ \\
\hline Score 2 & $9(17.6)$ \\
\hline Score 3 & $6(11.8)$ \\
\hline Score 4 & $21(41.2)$ \\
\hline \multicolumn{2}{|c|}{ Malignant transformation } \\
\hline Yes & $24(47.1)$ \\
\hline No & $27(52.9)$ \\
\hline
\end{tabular}

SD, standard deviation; LGD, low grade dysplasia; HGD, high grade dysplasia; BM, buccal mucosa; FOM, floor of the mouth.

However, 23 and $47 \%$ of patients with OPLs with LGD exhibited the expression of HuR and podoplanin, respectively. No significant association between protein expression and age, gender or site of the lesion was observed.

Median follow-up times were 43 months for the patients that did not develop an OSCC. During the follow-up period, 24/51 patients (47\%; 18 female and 6 male), developed OSCC. Malignant transformation occurred at a median of 55 months subsequent to diagnosis with a premalignant lesion. The estimated time for malignant transformation was 69 months from the primary diagnosis for $50 \%$ of the patients (Fig. 3). The calculated annual transformation rate was $8.7 \%$.

$H u R$ and podoplanin expression and the risk of oral cancer. One of the primary aims of the present study was to

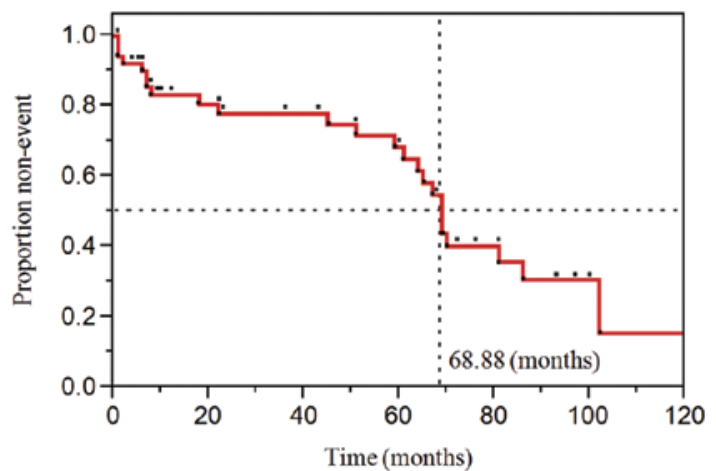

Figure 3. Kaplan-Meier curve for overall survival of all patients with oral preneoplastic lesions. A total of $50 \%$ of the cases experienced malignancy 69 months later, resulting in an annual transformation rate of $8.7 \%$. An 'event' is defined as malignant transformation.

determine whether protein expression (HuR and podoplanin) in a premalignant lesion is a feasible parameter to predict the patient's clinical outcome in terms of malignant transformation. Therefore, in order to estimate the time to malignant transformation of OPL, the OCFS time was assessed by the Kaplan-Meier method, using clinicopathological factors and HuR and podoplanin expression. In this analysis, HuR and podoplanin expression and the grade of dysplasia were established to be significant indicators using the log-rank test. All findings are summarized in Table III and presented in Figs. 4 and 5. Patients demonstrating HuR (Fig. 4A) and podoplanin (Fig. 4B) expression experienced a significantly increased oral cancer incidence compared with patients that did not express the proteins $(\mathrm{P}=0.001$ and $\mathrm{P}=0.006$, respectively). The results showed that 58 and $75 \%$ of the OPLs expressing HuR and podoplanin became malignant, respectively, and the expression demonstrates a stepwise pattern of malignant transformation (Fig. 4C and D; Table III). In addition, a statistically significant association existed between the histopathological grade and the risk of progression from dysplasia to oral cancer $(\mathrm{P}=0.027)$ (Fig. $5 \mathrm{~A})$. In order to determine whether protein expression augments oral cancer risk, the combination of HuR and podoplanin expression and the degree of dysplasia in OPLs was also analyzed. The lesions that expressed the two proteins turned malignant in a significantly shorter period, regardless of histopathology $(\mathrm{P}=0.005)$ (Fig. 5B; Table III).

To evaluate the oral cancer risk in patients with OPLs, clinicopathological parameters and HuR and podoplanin expression were analyzed using the Cox proportional hazards model (Table IV). For the univariate analysis, the expression of HuR (HR, 4.99; 95\% CI, 1.93-14.01; P=0.001), podoplanin (HR, 4.01; 95\% CI, 1.49-12.92; $\mathrm{P}=0.005)$ and the grade of dysplasia (HR, 2.75; 95\% CI, 1.11-7.32; $\mathrm{P}=0.029$ ) were significantly associated with an increased risk of malignant transformation. A multivariate analysis was performed in order to assess the factors that had a significant impact on the OCFS time in the univariate analysis, including histology, podoplanin and HuR expression. For the multivariate analysis, the adjusted HR for malignant transformation was 2.93 for HuR expression $(95 \%$ CI, 0.98-10.34; $\mathrm{P}=0.055)$. Notably, when the histology and coexpression of HuR and podoplanin were considered as cofactors, 
Table II. Association between HuR and podoplanin expression and clinicopathological parameters.

\begin{tabular}{|c|c|c|c|c|c|c|c|}
\hline \multirow[b]{2}{*}{ Characteristics } & \multirow[b]{2}{*}{$\begin{array}{c}\text { No. of } \\
\text { patients }\end{array}$} & \multicolumn{2}{|c|}{ HuR expression } & \multirow[b]{2}{*}{ P-value } & \multicolumn{2}{|c|}{ Podoplanin expression } & \multirow[b]{2}{*}{ P-value } \\
\hline & & $\begin{array}{l}\text { Not expressed } \\
\text { (level 1), } \\
\text { n }(\%)\end{array}$ & $\begin{array}{c}\text { Expressed } \\
\text { (levels } 2 \& 3) \\
\text { n }(\%)\end{array}$ & & $\begin{array}{c}\text { Not expressed } \\
\text { (scores } 0-1) \\
\mathrm{n}(\%)\end{array}$ & $\begin{array}{c}\text { Expressed } \\
\text { (scores 2-4), } \\
\text { n }(\%)\end{array}$ & \\
\hline All patients & 51 & $23(45)$ & $28(55)$ & & $15(29)$ & $36(71)$ & \\
\hline Age, years & & & & 0.676 & & & 0.702 \\
\hline Mean & & $72 \pm 12$ & $70 \pm 09$ & & $72 \pm 13$ & $71 \pm 09$ & \\
\hline Median & & 75 & 71 & & 75 & 71 & \\
\hline Max/Min & & $90 / 50$ & $82 / 55$ & & $90 / 50$ & $82 / 54$ & \\
\hline Gender & & & & 0.349 & & & 0.067 \\
\hline Female & 39 & 19 (49) & $20(51)$ & & $14(36)$ & $25(64)$ & \\
\hline Male & 12 & $4(33)$ & $8(67)$ & & $1(8)$ & $11(92)$ & \\
\hline Site & & & & 0.497 & & & 0.393 \\
\hline Tongue & 21 & $8(38)$ & $13(62)$ & & $5(24)$ & $16(76)$ & \\
\hline Gingiva & 8 & $3(38)$ & $5(63)$ & & $2(25)$ & $6(75)$ & \\
\hline $\mathrm{BM}$ & 14 & $9(64)$ & $5(36)$ & & $6(43)$ & $8(57)$ & \\
\hline FOM & 4 & $1(25)$ & $3(75)$ & & $0(0)$ & $4(100)$ & \\
\hline Others & 4 & $2(50)$ & $2(50)$ & & $2(50)$ & $2(50)$ & \\
\hline Dysplasia & & & & 0.001 & & & 0.009 \\
\hline LGD & 17 & $13(76)$ & $4(23)$ & & $9(53)$ & $8(47)$ & \\
\hline HGD & 34 & $10(29)$ & $24(71)$ & & $6(18)$ & $28(82)$ & \\
\hline
\end{tabular}

P-values of $<0.05$ were considered to indicate a statistically significant difference. HuR, ELAV like RNA binding protein 1; LDG, low grade dysplasia; HGD, high grade dysplasia; BM, buccal mucosa; FOM, floor of the mouth.
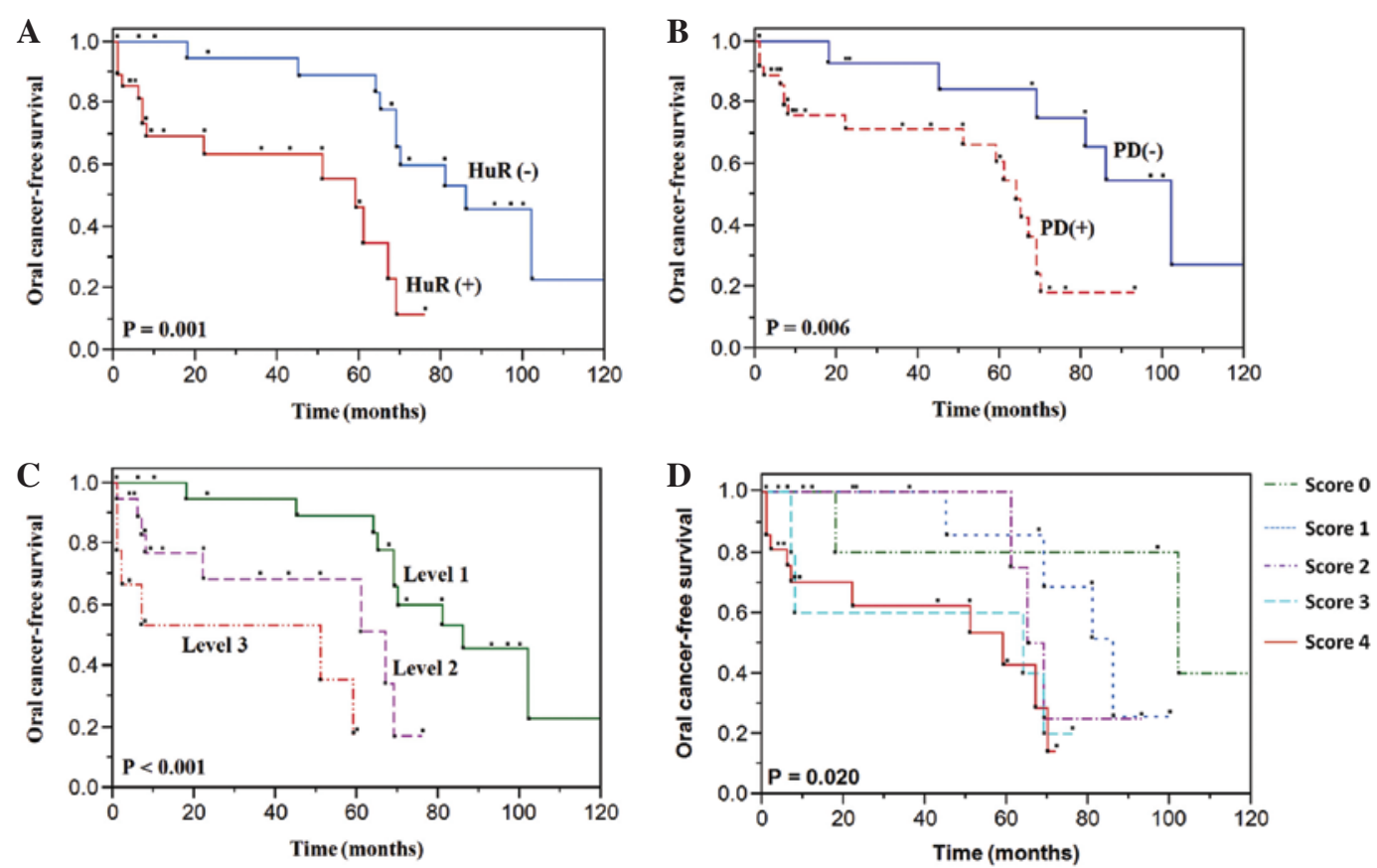

Figure 4. Kaplan-Meier oral cancer-free survival curves, by (A) HuR negativity and positivity, (B) PD negativity and positivity, (C) HuR levels and (D) PD scores. HuR, ELAV like RNA binding protein 1; PD, podoplanin.

the risk of the malignant transformation of OPLs was considerably increased compared with OPLs without coexpression and histology (HR, 5.79; 95\% CI, 1.64-23.59; $\mathrm{P}=0.005)$.

\section{Discussion}

The present study aimed to determine the usefulness of HuR 
Table III. Univariate survival analysis by significant prognostic factors using Kaplan-Meier modeling.

\begin{tabular}{|c|c|c|c|c|c|}
\hline Prognostic factors & $\begin{array}{l}\text { No. of } \\
\text { patients }\end{array}$ & $\begin{array}{l}\text { Patients without } \\
\text { malignant } \\
\text { transformation, } \\
\mathrm{n}(\%)\end{array}$ & $\begin{array}{c}\text { Patients with } \\
\text { malignant } \\
\text { transformation, } \\
\text { n }(\%)\end{array}$ & $\begin{array}{l}\text { Mean survival time, } \\
\text { months, standard } \\
\text { error }(95 \% \mathrm{CI})\end{array}$ & P-value \\
\hline $\mathrm{HuR}$ & & & & & 0.001 \\
\hline Negative (level 1) & 23 & $13(57)$ & $10(43)$ & $85.8,7.1(72.1-99.7)$ & \\
\hline Positive (levels 2-3) & 28 & $14(50)$ & $14(50)$ & $43.8,6.1(32.1-55.5)$ & \\
\hline Podoplanin & & & & & 0.006 \\
\hline Negative (score 0-1) & 15 & $9(60)$ & $6(40)$ & $89.3,8.9(71.7-106.8)$ & \\
\hline Positive (score 2-4) & 36 & $18(50)$ & $18(50)$ & $53.1,5.9(41.5-64.8)$ & \\
\hline HuR level & & & & & 0.000 \\
\hline Level 1 & 23 & $13(57)$ & $10(43)$ & 85.8, 7.1 (71.9-99.7) & \\
\hline Level 2 & 19 & $11(58)$ & $8(42)$ & $49.9,7.2(35.8-63.9)$ & \\
\hline Level 3 & 9 & $3(33)$ & $6(67)$ & $31.6,6.4(54.9-80.1)$ & \\
\hline PDS & & & & & 0.020 \\
\hline PDS-0 & 5 & $3(60)$ & $2(40)$ & $92.4,17.4(58.3-126.5)$ & \\
\hline PDS-1 & 10 & $6(60)$ & $4(40)$ & 79.9, $7.0(66.2-93.7)$ & \\
\hline PDS-2 & 9 & $6(67)$ & $3(33)$ & $72.0,6.2(59.8-84.2)$ & \\
\hline PDS-3 & 6 & $2(33)$ & $4(67)$ & $44.8,13.7(17.9-71.7)$ & \\
\hline PDS-4 & 21 & $10(48)$ & $11(52)$ & $43.3,6.9(29.8-56.8)$ & \\
\hline Dysplasia & & & & & 0.027 \\
\hline LGD & 17 & $8(47)$ & $9(53)$ & $78.4,10.2(58.5-98.3)$ & \\
\hline HGD & 34 & $19(56)$ & $15(44)$ & $53.9,6.2(41.7-66.1)$ & \\
\hline $\begin{array}{l}\text { HuR and PD expression } \\
\text { and histopathology }\end{array}$ & & & & & 0.005 \\
\hline $\mathrm{HuR}^{+} \mathrm{PD}^{+} \mathrm{HGD}$ & 23 & $12(52)$ & $11(48)$ & $45.5,6.3(33.1-58.1)$ & \\
\hline $\mathrm{HuR}^{+} \mathrm{PD}^{+} \mathrm{LGD}$ & 4 & $1(25)$ & $3(75)$ & $21.7,15.7(0.0-52.5)$ & \\
\hline $\mathrm{HuR}^{-} \mathrm{PD}^{-} \mathrm{HGD}$ & 5 & $3(60)$ & $2(40)$ & $57.7,17.9(22.5-92.9)$ & \\
\hline $\mathrm{HuR}^{-} \mathrm{PD}^{-} \mathrm{LGD}$ & 9 & $5(56)$ & $4(44)$ & $98.2,7.0(84.5-112.0)$ & \\
\hline Otherwise $^{\mathrm{a}}$ & 10 & $6(60)$ & $4(40)$ & $76.1,5.1(65.7-85.6)$ & \\
\hline
\end{tabular}

${ }^{\mathrm{a}}$ Otherwise: $\mathrm{Hu}^{+} \mathrm{PD}^{-} \mathrm{HGD}, \mathrm{HuR}^{+} \mathrm{PD}^{-} \mathrm{LGD}, \mathrm{HuR}^{-} \mathrm{PD}^{+} \mathrm{HGD}$ or HuR $\mathrm{PD}^{+}$LGD. CI, confidence interval; HuR, ELAV like RNA binding protein 1; PDS, podoplanin score; LGD, low grade dysplasia; HGD, high grade dysplasia; PD, podoplanin.
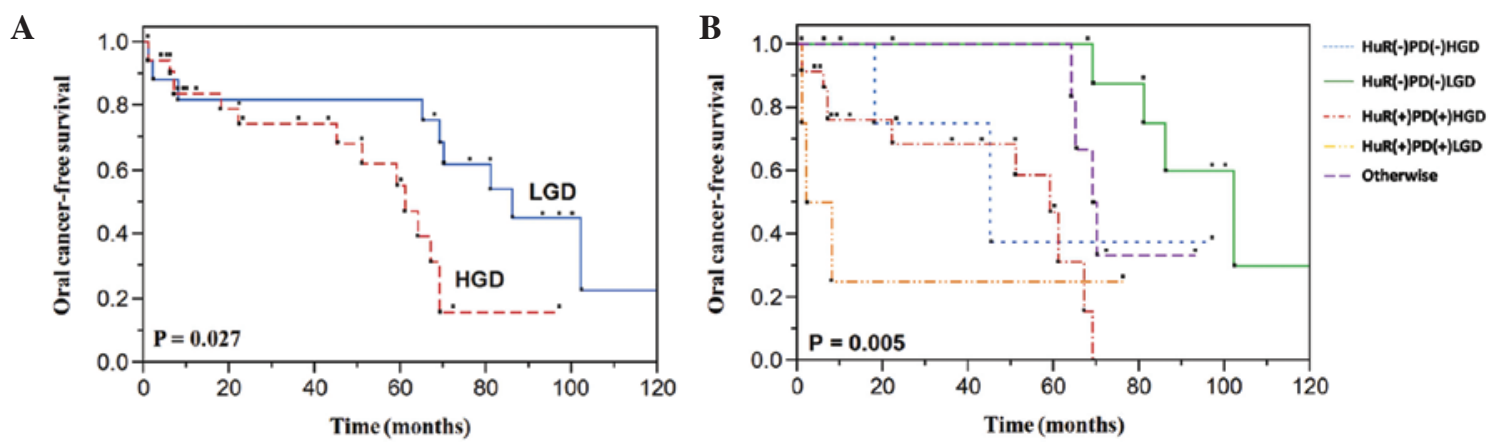

Figure 5. Kaplan-Meier oral cancer-free survival curves, by (A) degree of dysplasia and (B) HuR and PD expression and histology. HuR, ELAV like RNA binding protein 1; PD, podoplanin; LGD, low grade dysplasia; HGD, high grade dysplasia.

and podoplanin in predicting the risk of malignant transformation in patients with OPL. Dysplasia and protein expression (HuR and podoplanin) were indicated to be significant predictors for malignant transformation in OPL (Table III).
Increasing numbers of studies regarding the human ELAV-like protein HuR are being undertaken, as HuR regulates the mRNA stability of numerous growth-promoting genes (8). As increased cytoplasmic HuR expression has 
Table IV. Cox proportional hazard regression models in estimating cancer development.

\begin{tabular}{|c|c|c|c|}
\hline Characteristics & Hazard ratio & $95 \% \mathrm{CI}$ & P-value \\
\hline \multicolumn{4}{|l|}{ Univariate analysis } \\
\hline Age, $\leq 71$ vs. $>71$ years & 1.18 & $0.51-2.65$ & 0.696 \\
\hline Sex, male vs. female & 1.38 & $0.49-3.36$ & 0.051 \\
\hline Histology, HGD vs. LGD & 2.75 & $1.11-7.32$ & 0.029 \\
\hline Podoplanin, expressed vs. not expressed & 4.01 & $1.49-12.92$ & 0.005 \\
\hline HuR, expressed vs. not expressed & 4.99 & $1.93-14.01$ & 0.001 \\
\hline \multicolumn{4}{|c|}{ Multivariate analysis, histology, podoplanin and HuR } \\
\hline Histology, HGD vs. LGD & 1.45 & $0.52-4.36$ & 0.486 \\
\hline Podoplanin, expressed vs. not expressed & 2.06 & $0.55-8.01$ & 0.283 \\
\hline HuR, expressed vs. not expressed & 2.93 & $0.98-10.34$ & 0.055 \\
\hline \multicolumn{4}{|c|}{ Multivariate analysis, histology, coexpression of podoplanin and HuR } \\
\hline Histology, HGD vs. LGD & 1.47 & $0.52-4.44$ & 0.477 \\
\hline \multicolumn{4}{|l|}{ Podoplanin and HuR } \\
\hline Both & 5.79 & $1.64-23.59$ & 0.005 \\
\hline Either & 1.78 & $0.41-7.31$ & 0.424 \\
\hline
\end{tabular}

P-values of $<0.05$ were considered to indicate a statistically significant difference. CI, confidence interval; HGD, high grade dysplasia; LGD, low grade dysplasia; HuR, ELAV like RNA binding protein 1.

been detected in human malignant tumors, a deregulated HuR pathway has been suggested to have implications in cancer biology, by promoting the abnormal expression of several proteins (10). Cytoplasmic HuR expression has been indicated in colon, ovary, breast, salivary gland, uterus, larynx and prostate malignancies, and has been postulated to contribute to the cancerous malignant phenotype (19-23). In agreement with previous studies, the present study also indicated that HuR may be used as a diagnostic marker for oral cancer (24). A recently published study (18) reported the significant role of HuR in assessing the risk of malignant transformation in patients with oral verrucous lesions. The present study reported that HuR expression in OPL was associated with an increased risk of malignant transformation $(\mathrm{P}<0.05)$.

Podoplanin as a cancer stem cell marker has been reported to be expressed in approximately $90 \%$ of OSCCs and restricted to the invasive front of squamous cell carcinoma (15). Podoplanin was also reported to be overexpressed in the basal cell layers of certain hyperplastic and dysplastic regions located adjacent to OSCC (25-27). Kawaguchi et al (14) reported podoplanin as a marker of malignant transformation in oral leukoplakia and other oral precancerous lesions. The present results are in agreement with Kawaguchi's observation, suggesting that podoplanin expression in OPL is associated with an increased risk of malignant transformation $(\mathrm{P}=0.006)$. Other studies also support that podoplanin has a relevant role in early oral tumorigenesis, even considering that podoplanin expression alone may not be sufficient to promote carcinogenesis (12-15).

The present study included podoplanin and performed immunohistochemical staining of HuR and podoplanin to evaluate oral cancer risk in patients with OPL. In the univariate analysis, HuR and podoplanin expression was associated with a 4.99-fold and 4.01-fold increased risk of malignant transformation, respectively $(\mathrm{P}<0.05)$. HuR and podoplanin expression in OPL showed a significant impact on OCFS, with a decreasing 5-year OCFS rate of $100 \%$, for patients with no expression, and of 35 and $42 \%$ for patients with increased HuR (level 3) and podoplanin (score 4) expression, respectively $(\mathrm{P}<0.05)$ (Fig. $4 \mathrm{C}$ and $\mathrm{D}$; Table III). Additionally, in the multivariate analysis, a significant difference in the coexpression of HuR and podoplanin and histological features was indicated to be associated with the malignant transformation of OPL $(\mathrm{P}<0.05)$. Overall, these data support the potential importance of HuR and podoplanin in oral carcinogenesis and also suggest that each may be used as biomarkers for evaluating malignant transformation risk in oral premalignancy. However, contrary to the previous findings, $10(42 \%)$ and 6 (25\%) patients without HuR and podoplanin expression, respectively, developed cancers in the present study (Table III), although development was delayed compared with patients that expressed $\mathrm{HuR}$ and podoplanin. The plausible reasons behind the delayed but cancerous transformation may be attributed to the lesions being biopsied prior to the abnormality occurring, or to the cancers originating from lesions that were not clinically visible at the time of biopsy and therefore remained unexamined. Another possibility is that the biopsies were taken from other clonal sites compared with the sites from which the cancers eventually developed (14).

Although there is an almost general agreement that the rate of malignant transformation increases with the severity of the dysplasia, certain studies did not observe a significant association between epithelial dysplasia and malignant transformation (28). In addition, substantial interobserver and intraobserver variation exists, in terms of evaluating the presence and severity of epithelial dysplasia $(29,30)$. In the samples 
in the present study, $9(53 \%)$ of 17 cases of LGD developed OSCC during the follow-up period. Conversely, 19 (56\%) of 34 cases showing severe dysplasia did not develop OSCC. The combination of HuR and podoplanin expression and the degree of dysplasia in association with malignant transformation was also analyzed. As shown in Table III, $>50 \%$ of the cases that underwent malignant transformation expressed the two proteins regardless of histopathology $(\mathrm{P}=0.005)$. Notably, the tumors of only $2(8 \%)$ patients with HGD, who did not express the proteins, became malignant. Surprisingly, the tumors of patients with LGD that demonstrated the expression of the two proteins turned malignant within a shorter period of time compared with patients with HGD. In the univariate analysis, histology was one of the significant factors for malignant transformation; however, the HR was 1.6-fold decreased compared with the protein expression. Notably, when histology and the coexpression of HuR and podoplanin were considered as cofactors, the risk of OPL malignant transformation was considerably increased compared with OPL without coexpression (HR=5.79; 95\% CI, 1.64-23.59; $\mathrm{P}=0.005$ ) and histology. This finding suggests that the coexpression of the two biomarkers may be more informative compared with the histological examination alone. Therefore, immunohistochemical staining of $\mathrm{HuR}$ and podoplanin may contribute to augmenting the predictability and reliability in cancer risk assessment of OPL, in association with a histopathological assessment of epithelial dysplasia.

Cancer of the oral cavity results in severe morbidity, compromised quality of life and short overall survival. Therefore, there is a strong need to understand oral carcinogenesis and to establish accurate and reliable predictors of oral cancer risk (1-3,31). To the best of our knowledge, the present study is the first to investigate the role of HuR in oral cancer risk assessments and to evaluate the combined expression of HuR and podoplanin in patients with OPL. The present data showed that patients with OPL that demonstrate the expression of HuR and podoplanin were significantly associated with malignant transformation risk; consequently, these patients are recommended for careful follow-up. In summary, HuR and podoplanin may be used as biomarkers for the risk assessment of oral malignant transformation in patients with OPL. However, additional studies are required in order to fully define the functional role of these biomarkers in oral cancer initiation and disease progression.

\section{Acknowledgements}

The author was a recipient of the Iwadare Scholarship. The authors thank Dr Yoichi M.ITO (Department of Biostatistics, Hokkaido University Graduate School of Medicine) for statistical support, and thank Mr. Yohei Murayama and Ms. Tomomi Takahashi (Support Section for Education and Research, Hokkaido University Graduate School of Dental Medicine) for the technical assistance.

\section{References}

1. Ganly I, Patel S and Shah J: Early stage squamous cell cancer of the oral tongue - clinicopathologic features affecting outcome. Cancer 118: 101-111, 2012.

2. Zini A, Czerninski R and Sgan-Cohen HD: Oral cancer over four decades. Epidemiology, trends, histology and survival by anatomical sites. J Oral Pathol Med 39: 299-305, 2010.
3. Warnakulasuriya S: Living with oral cancer: Epidemiology with particular reference to prevalence and life-style changes that influence survival. Oral Oncol 46: 407-410, 2010.

4. Silverman S Jr, Gorsky M and Lozada F: Oral leukoplakia and malignant transformation. A follow-up study of 257 patients. Cancer 53: 563-568, 1984.

5. Papadimitrakopoulou VA, Hong WK, Lee JS, Martin JW, Lee JJ, Batsakis JG and Lippman SM: Low-dose isotretinoin versus beta-carotene to prevent oral carcinogenesis: Long-term follow-up. J Natl Cancer Inst 89: 257-258, 1997.

6. Fan XC and Steitz JA: HNS, a nuclear-cytoplasmic shuttling sequence in HuR. Proc Natl Acad Sci USA 95: 15293-15298, 1998.

7. Rebane A, Aab A and Steitz JA: Transportin 1 and 2 are redundant nuclear import factors for hnRNP A1 and HuR. RNA 10: 590-599, 2004.

8. Brennan CM, Gallouzi IE and Steitz JA: Protein ligands to HuR modulate its interaction with target mRNAs in vivo. J Cell Biol 151: 1-14, 2000.

9. Erkinheimo TL, Lassus H, Sivula A, Sengupta S, Furneaux H, Hla T, Haglund C, Butzow R and Ristimäki A: Cytoplasmic HuR expression correlates with poor outcome and with cyclooxygenase 2 expression in serous ovarian carcinoma. Cancer Res 63: 7591-7594, 2003.

10. Denkert C, Weichert W, Pest S, Koch I, Licht D, Köbel M, Reles A, Sehouli J, Dietel M and Hauptmann S: Overexpression of the embryonic-lethal abnormal vision-like protein HuR in ovarian carcinoma is a prognostic factor and is associated with increased cyclooxygenase 2 expression. Cancer Res 64: 189-195, 2004

11. Kahn HJ and Marks A: A new monoclonal antibody, D2-40, for detection of lymphatic invasion in primary tumors. Lab Invest 82: 1255-1257, 2002.

12. Inoue H, Miyazaki Y, Kikuchi K, Yoshida N, Ide F, Ohmori Y, Tomomura A, Sakashita H and Kusama K: Podoplanin expression during dysplasia-carcinoma sequence in the oral cavity. Tumor Biol 33: 181-194, 2012.

13. Saintigny P, El-Naggar AK, Papadimitrakopoulou V, Ren H, Fan YH, Feng L, Lee JJ, Kim ES, Hong WK, Lippman SM and Mao L: DeltaNp63 over expression, alone and in combination with other biomarkers, predicts the development of oral cancer in patients with leukoplakia. Clin Cancer Res 15: 6284-6291, 2009.

14. Kawaguchi H, El-Naggar A, Papadimitrakopoulou V, Ren H, Fan YH, Feng L, Lee JJ, Kim E, Hong WK, Lippman SM and Mao L: Podoplanin: A novel marker for oral cancer risk in patients with oral premalignancy. J Clin Oncol 26: 354-360, 2008.

15. Wicki A and Christofori G: The potential role of podoplanin in tumour invasion. Br J Cancer 96: 1-5, 2007.

16. Martín-Villar E, Scholl FG, Gamallo C, Yuritta MM, Muñoz-Guerra M, Cruces J and Quintanilla M: Characterization of human PA2.26 antigen (T1alpha-2, podoplanin), a small membrane mucin induced in oral squamous cell carcinomas. Int J Cancer 113: 899-910, 2005.

17. Mithani SK, Mydlarz WK, Grumbione FL, Smith IM and Califano JA: Molecular genetics of premalignant oral lesions. Oral Diseases 13: 126-133, 2007.

18. Habiba U, Kitamura T, Yanagawa-Matsuda A, Hida K, Higashino F, Ohiro Y, Totsuka Y and Shindoh M: Cytoplasmic expression of HuR may be a valuable diagnostic tool for determining the potential for malignant transformation of oral verrucous borderline lesions. Oncol Rep 31: 1547-1554, 2014.

19. Cho NP, Han HS, Soh Y, Lee KY and Son HJ: Cytoplasmic HuR over-expression is associated with increased cyclooxygenase-2 expression in laryngeal squamous cell carcinomas. Pathology 39: 545-550, 2007.

20. Cho NP, Han HS, Soh Y and Son HJ: Overexpression of cyclooxygenase-2 correlates with cytoplasmic HuR expression in salivary mucoepidermoid carcinoma but not in pleomorphic adenoma. J Oral Pathol Med 36: 297-303, 2007.

21. Heinonen M, Bono P, Narko K, Chang SH, Lundin J, Joensuu H, Furneaux H, Hla T, Haglund C and Ristimäki A: Cytoplasmic $\mathrm{HuR}$ expression is a prognostic factor in invasive ductal breast carcinoma. Cancer Res 65: 2157-2161, 2005.

22. Niesporek S, Kristiansen G, Thoma A, Weichert W, Noske A, Buckendahl AC, Jung K, Stephan C, Dietel M and Denkert C: Expression of the ELAV-like protein HuR in human prostate carcinoma is an indicator of disease relapse and linked to COX-2 expression. Int J Oncol 32: 341-347, 2008. 
23. Gallouzi IE and Steitz JA: Delineation of mRNA export pathways by the use of cell-permeable peptides. Science 294: 1895-1901, 2001.

24. Hasegawa H, Kakuguchi W, Kuroshima T, Kitamura T, Tanaka S, Kitagawa Y, Totsuka Y, Shindoh M and Higashino F: HuR is exported to the cytoplasm in oral cancer cells in a different manner from that of normal cells. Br J Cancer 100: 1943-1948, 2009.

25. Yuan P, Temam S, El-Naggar A, Zhou X, Liu DD, Lee JJ and Mao L: Overexpressin of podoplanin in oral cancer and its association with poor clinical outcome. Cancer 107: 563-569, 2006.

26. Kreppel M, Scheer M, Drebber U, Ritter L and Zöller JE: Impact of podoplanin expression in oral squamous cell carcinoma: Clinical and histopathologic correlations. Virchows Arch 456: 473-482, 2010.

27. Bolzoni Villaret A, Schreiber A, Facchetti F, Fisogni S, Lonardi S, Lombardi D, Cocco D, Redaelli de Zinis LO and Nicolai P: Immunostaining patterns of CD31 and podoplanin in previously untreated advanced oral/oropharyngeal cancer: Prognostic implications. Head neck 32: 786-792, 2010.
28. Holmstrup P, Vedtofte P, Reibel J and Stoltze K: Long term treatment outcome of oral premalignant lesions. Oral Oncol 42: 461-474, 2006.

29. Karrabul A, Reibel J, Therkildsen MH, Praetorius F, Nielsen HW and Dabelsteen E: Observer variability in the histologic assessment of oral premalignant lesions. J Pathol Med 24: 198-200, 1995.

30. Abbey LM, Kaugars GE, Gunsolley JC, Burns JC, Page DG, Svirsky JA, Eisenberg E, Krutchkoff DJ and Cushing M: Intraexaminer and interexaminer reliability in diagnosis of oral epithelial dysplasia. Oral Surg Oral Med Oral Pathol Oral Radiol Endod 80: 188-191, 1995.

31. de Vicente JC, Rodrigo JP, Rodriguez-Santamarta T, Lequerica-Fernández $\mathrm{P}$, Allonca E and Garcia-Pedrero JM: Podoplanin expression in oral leukoplakia: Tumorigenic role. Oral Oncol 49: 598-603, 2013. 